\title{
An Efficient Encryption-Then-Compression System using Asymmetric Numeral Method
}

\author{
P.Sridevi ${ }^{1}$ and Dr. J. Suguna ${ }^{2}$ \\ ${ }^{1}$ Research Scholar, Department of Computer Science, Vellalar College for Women Tamilnadu, India \\ sridevi@vcw.ac.in \\ ${ }^{2}$ Associate Professor, Department of Computer Science, Vellalar College for Women Tamilnadu, India \\ sugunajravi@yahoo.co.in
}

\begin{abstract}
Image compression is an important task in the field of image processing and it is an essential process in digital world to reduce the memory for storage and processing of the images. Many practical applications need compression and security. Encryption is a security control and widely used in many computer applications to provide protection for data. The images are encrypted before compression to give high level security and drawn much attention in several applications like data transferring, medical, navy and military operations. This paper has proposed an efficient image Encryption and Then Compression system (ETC) using Asymmetric Numeral Method (ANM) for compressing the images. The proposed ANM coder is compared with the existing Huffman and Arithmetic coder. The comparative analysis will be carried out for different size of images and results are illustrated that the proposed system is efficient in terms of compression ratio (Bits per Pixel) and computation time.
\end{abstract}

Keywords - Image Encryption, Lossless Compression, Security, Asymmetric Numerical Method and Prediction Error Clustering.

\section{INTRODUCTION}

In this digital era, huge amount of data is transferred every second and the use of digital images are also increased. Digital images require more time for transmission because of their larger size. Hence, it is important to compress the images in order to improve the processing. An important goal of image compression is to reduce the bit rate for storage without altering the image quality. Image compression is the process of minimizing the size without degrading the quality of the image. The reduction in file size allows more images to be stored in a given memory space. It also reduces the time required for images to be sent over the Internet.

Image compression is of two types: lossy and lossless. In lossy compression, original image is not identical to decompressed image that means there is some loss. Lossy compression is generally used for natural and photography images. Block Truncation Coding and Transform Coding are lossy compression technique. In lossless compression original image and decompressed image are equal, that means there is no loss. Lossless compression provides better compression for highly sensitive applications. Run Length Coding, Huffman Coding, Lempel Ziv Coding and Arithmetic Coding are lossless compression technique. A lossless compression technique is preferred for all purposes and especially for medical imaging and technical drawings.

Encryption before compression brings more attention in recent years due to secure transmission in many applications. In Encryption-Then-Compression (ETC) technique, compression has to be conducted after encryption to enhance secure transmission. For fast transmission and protection of information, ETC is carried out in two steps [5]. The first step is an operation of encryption to modify the information and make them unreadable format [10]. The second step is an operation of compression in which the size of information to be transmitted is reduced by removing redundant information.

The encryption techniques are of two categories, they are Symmetric and Asymmetric encryption techniques. Symmetric encryption is the simplest encryption where the same key is used for encryption and decryption. In symmetric encryption sender and receiver use a shared key to encrypt or decrypt the data. The only problem with this technique is that if the key is known to others the entire system is collapsed. In Asymmetric encryption technique both sender and receiver use a separate key to encrypt and decrypt the data [7]. Asymmetric encryption uses two keys to encrypt a plain text. One of the key is known as the private key and the other is known as the public key. The private key is kept secret by the owner and the public key is either shared among authorized recipients or made available to the public at large.

The aim of ETC is to reduce the image size and provide sufficient level of security with minimum computation time. Reducing the computation time is very desirable especially in constrained communications like real-time networking, high-definition delivery and mobile communications with limited computational power devices. ETC system is used to protect the file contents, which could contain confidential and secret informations. 
The rest of this paper is organized as follows. Section II explains the related researches briefly. Section III provides the details of proposed ETC system, where Asymmetric Numerical Method [ANM] is considered for image compression. Section IV presents the performance analysis of ANM. Section V provides the experimental results and their discussions. Section VI concludes the research work.

\section{RELATED WORK}

Johnson et. al [6] proposed a scheme to compress an encrypted image by using performance of theoretical results and showed that the stream cipher encrypted data is compressible through the use of coding with information principles, without compromising either the compression efficiency or the information-theoretic security. In addition to theoretical findings, the author proposed practical algorithms for lossless compression on encrypted binary images by using Low-Density Parity-Check (LDPC) channel code.

Lazzeretti and Barni [8] presented several methods for lossless compression of encrypted grayscale or color images. The author discussed about bitplanes, prediction error, spatial and cross-plane correlation among pixels and obtained best results by transforming color images in an approximated $\mathrm{YCbCr}$ domain.

Zhang et.al [11] discusssed a scalable coding framework of encrypted images via multi-resolution construction. The author implemented an encryption of modulo- 256 addition with series of pseudorandom numbers derived from a secret key, designed an image encryption scheme via pixel-domain permutation and demonstrated that the encrypted file can be efficiently compressed by discarding the excessively rough and fine information of coefficients in the transform domain.

The encoded bitstreams are made up of a quantized encrypted subimage with multiple-resolution construction and suggested compressive sensing (CS) mechanism to compress an encrypted image.

Jiantao Zhou et.al [5] presented a design of an efficient encryption then-compression system. In this approach a permutation based image encryption method was conducted over the prediction error domain. The arithmetic coding approach efficiently compress the encrypted image and it provides reasonably high level of security, but the compression approach applied to encrypted images is not good in terms of compression efficiency.

Xinpeng Zhang et.al [9] proposed a novel scheme of lossy compression for encrypted gray images. In the encryption phase, the original image is decomposed as set of coefficients by a secret orthogonal transform and then compressed by a linear operation. The method achieved good result in reconstruction of images.

Jarek Duda [1] proposed Asymmetric Numeral System (ANS) approach to entropy coding and combined the compression ratio of arithmetic coding with the speed of huffman coding. In this paper single natural number is used as the state, instead of two to represent a range. The author discussed about many other variants like uniform asymmetric binary system, encoding finite state automaton and probability distribution of states.

\section{Proposed SYSTEM}

The three key components for proposed ETC system are image encryption, image compression and the sequential decryption and decompression. ETC is the method for securing the data, which is very important for online transactions, data transfer through network, navy operations and medical image processing. The existing work addressed encryption and compression of image using Arithmetic Coder and Huffman Coder [5]. Arithmetic Coder results in good compression ratio with larger computation time. Huffman Coder is fast but it gives low compression rate. Asymmetric Numerical Method [ANM] is used to overcome the above issues. ANM is very efficient and faster decoding method because of its simple design.

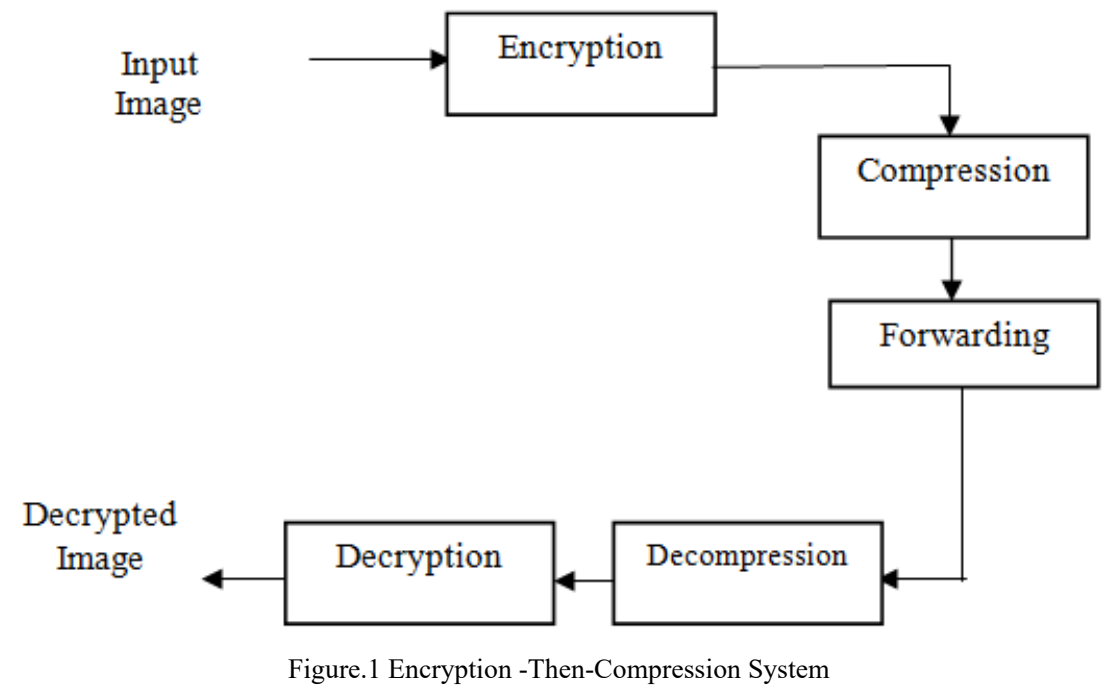




\section{A. Image Encryption}

Design of encryption process operated over the prediction error domain in existing system, for each pixel I (i, $j$ ) of the image I is to be encrypted, a prediction Ip $(i, j)$ is first made by using an image predictor. The prediction error can take any values in the range $[-255,255]$ for eight bit images and prediction error associated with image I can be calculated by

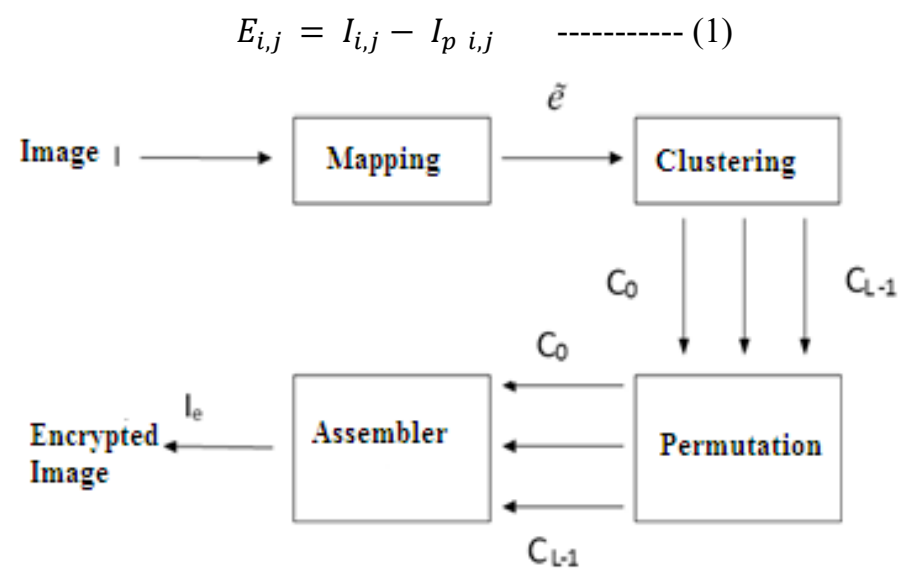

Figure.2 Image Encryption

In the ETC system the encryption is performed over the domain of the mapped prediction errors E (mp) and it divides the prediction errors into L clusters. Clustering operation is benefited in randomization and compression. The design of the cluster should consider the security and compression of encrypted image. Larger value of $\mathrm{L}$ provide higher level of security.

MOST SIGNIFICANT POSITION $x^{\prime}=x+s .2^{\wedge} m$
\begin{tabular}{|c|c|c|c|c|c|c|c|c|c|c|c|c|c|c|c|c|c|c|c|c|}
\cline { 3 - 3 } & \multicolumn{1}{|c|}{$x$} & 0 & 1 & 2 & 3 & 4 & 5 & 6 & 7 & 8 & 9 & 10 & 11 & 12 & 13 & 14 & 15 & 16 & 17 & 18 \\
\hline $\mathrm{S}=0$ & 0 & & 1 & & 2 & & 3 & & 4 & & 5 & & 6 & & 7 & & 8 & & 9 \\
\hline \multicolumn{2}{|c|}{$\mathrm{S}=0$} & \multicolumn{2}{|c|}{$\mathrm{S}=1$} \\
\hline 00 & 01 & 10 & 11 \\
\hline $\mathrm{S}=1$ & & 0 & & 1 & & 2 & & 3 & & 4 & & 5 & & 6 & & 7 & & 8 & \\
\hline
\end{tabular}

Range/arithmetic coding

\begin{tabular}{|l|l|l|l|}
\hline \multicolumn{2}{|c|}{$\mathrm{S}=0$} & \multicolumn{2}{c|}{$\mathrm{S}=1$} \\
\hline 00 & 01 & 10 & 11 \\
\hline
\end{tabular}

$x^{\star}=x / \operatorname{Pr}(s)$ Some asymmetric binary system for $\operatorname{Pr}(0)=1-\operatorname{Pr}(1)=0.7$ redefine even $/$ odd numbers $=$ change their densities

$5 \rightarrow 10 \mathrm{~S}=0,5 \rightarrow 11 \mathrm{~S}=1$

$5 \rightarrow 8 \mathrm{~S}=0,5 \rightarrow 16 \mathrm{~S}=1$

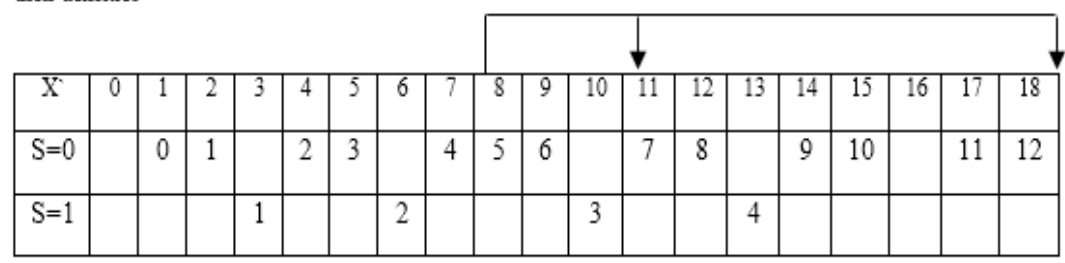

Figure.3. Asymmetric Numerical Method Encryption Algorithm

Step 1: Calculate all the mapped prediction errors $E(\mathrm{mp})$, of the image I .

Step 2: Split all the prediction errors into $L$ no of clusters $\left(C_{k}\right.$ for $\left.0 \leq k \leq L-1\right)$. $k$ is the cluster index.

Step 3: Resize the predicition errors in every $C_{k}$ into a two dimensional block which have four columns and $C_{k} / 4$ rows.

$\left|C_{k}\right|$ denotes the number of prediction errors in $C_{k}$

Step 4: Apply cyclic shift operations to each output of prediction error block and read the data in raster scan manner to obtain the permuted cluster $\mathrm{C}_{\mathrm{k}}$.

Step 5: The assembler concatenates all the permuted clusters $C_{k}$ for $0 \leq k \leq L-1$, and generate encrypted image.

$$
I_{e}=C_{0} C_{1} \ldots \ldots C_{L-1}
$$


in which each prediction error is represented by 8 bits. As the number of prediction errors equals that of the pixels, the file size before and after the encryption preserves.

Step 6: Pass encrypted image along with the length of each cluster $\left|C_{k}\right|$ for $0 \leq k \leq L-2$. The values of $\left|C_{k}\right|$ helps to divide encrypted image into L clusters.

\section{B. Asymmetric Numerical Method}

Asymmetric Numerical Method (ANM) is an entropy coding method used in compression to improve the compression performance. Entropy encoding is a lossless data compression scheme that is independent of the specific characteristics of the medium and also used to measure the amount of similarity between streams of data [8]. The Entropy coder are Huffman Coder (HC) and Arithmetic Coder (AC). Huffman coder is faster but uses approximate probabilities with powers of 2 which leads to relatively low compression rates. The arithmetic coder uses nearly exact probabilities with larger computational time [1]. ANM has the advantage to combine the compression ratio of arithmetic coder with speed of Huffman coder and it is used in image compression because of high performance and efficiency [2].

In standard binary numeral system, one can add bit of information from a digit $S €(0,1)$ to an existing number $X$ either to the most significant position $\left(x^{\prime} \rightarrow x^{+}+s 2^{\wedge} m\right)$ or to the least significant position $\left(x^{\prime} \rightarrow 2 x+s\right)$. In most significant position, new digit $\mathrm{S}$ can choose between ranges and two numbers [position of digit and range at given moment] are required to represent the current state. In least significant position, the current state is single natural number, this is the advantage of ANM. While in standard binary system, $\mathrm{X}$ becomes $\mathrm{X}$-th appearance of even (s $=0$ ) or odd ( $\mathrm{s}=1)$ number, so it redefines the splitting of $\mathrm{N}$ into even and odd number and uniformly distributed with different densities corresponding to symbol probability distribution[1]. From both approaches, half of the range is enough to represent 1 bit of information, so the current content is $\lg$ (size of range/size of sub range) bits of information. While encoding symbol of probability $p$, the size of sub range is multiplied by $p$, it increases informational content by $\lg (1 / \mathrm{p})$ bits. According to ANM, X contains $\lg (\mathrm{X})$ bits of information, informational content should increase to

$$
\lg (x)+\lg \left(\frac{1}{p}\right)=\lg \left(\frac{x}{p}\right) \text {--------- (3) }
$$

ANM add information in the least significant position. Its coding rule considered X as X-th appearance of S-th subset of natural numbers corresponding to currently encoded symbol. When uniform (symmetric) probability distribution of symbols are considered, In figure 3 , $\mathrm{X}$ value 5 represent 10 (if $\mathrm{s}=0$ ) and 5 represent 11 (if $\mathrm{s}=1$ ). ANM makes it optimal for asymmetric probability distribution of symbols. In ANM, even/odd division of natural numbers are replaced with division into subsets having densities corresponding to the assumed probability distribution. Here, $X$ value 5 represent 8 (if $s=0$ ) and 5 represent 16 (if $s=1$ ) is explained in figure 3 . The algorithm for proposed method is described below.

ANM Algorithm

Step 1: Pass Encrypted image to the channel provider.

Step 2: Asymmetric Numerical Method coding is applied.

Step 3: Compressed form of encrypted image is Send to the receiver where sequential decompression and decryption is done.

\section{Performance Analysis}

Performance analysis plays an important role in the design of encryption and compression algorithms. The performance of the proposed ANM compression algorithm is compared with that of the two existing algorithms namely Arithmetic coder and Huffman coder. Arithmetic coder is a lossless coding technique and it has a better compression ratio than Huffman coder. Arithmetic coder is based on the frequency of symbols and the whole data is represented by a fractional number between 0 and 1 . Optimality and flexibility is the main advantage of arithmetic coder. Arithmetic coder allows the transfer of large volume of data with limited resources with less reliability. The arithmetic coder uses nearly exact probabilities with larger computational time. A main disadvantage of arithmetic coder is its poor error resistance. If there is a single bit error in the codeword, the entire data become corrupted and may cause the retransmission of the entire data. Huffman coder is optimal and it is based on the frequency of occurrence of a data item (pixel in images). The principle is to use a lower number of bits to encode the data that occurs more frequently and directly translate a symbol into a bit sequence. Huffman coder is faster but uses approximate probabilities with powers of 2 which leads to relatively low compression rates. Huffman coder requires sorting of symbols to build prefix tree which is costly.

The proposed ANM is cheap and provide effective compression. ANM has the advantage to combine the compression ratio of arithmetic coder with the speed of Huffman coder and it is used in image compression because of high performance and efficiency [1] \& [3]. ANM performs faster decoding than Huffman coder. ANM coder can be implemented with less computational complexity. ANM is the best precise coder and more resistant to brute force attacks. ANM has a lower MSE and a higher PSNR. A lower value for MSE means lesser error and 
higher value of PSNR is good because it means that the ratio of signal to noise is higher. Here, the 'signal' is the original image, and the 'noise' is the error in reconstruction. The results proved that ANM is the best compression algorithm in terms of compression ratio, MSE, PSNR and computation time.

\section{EXPERIMENTAL RESULTS}

In this section the performance of the proposed Asymmetric Numerical Method (ANM) is evaluated and compared with the performance of Huffman coder and Arithmetic coder. Bits per pixel (BPP), Compression Ratio and computation time are the performance metrics considered for evaluation. The Experimental evaluation of proposed ETC is simulated by using MATLAB. The test set is composed of 100 images with various characteristics, 10 of which are used to display the result. Table 1 shows the comparison of proposed ANM with the existing Huffman and Arithmetic coder in terms of compression performance [B represents bytes]. ANM shows better performance for all the images and compression performance is increased from $8 \%$ to $12 \%$ for medical and satellite images.

Table 1 Compression Performance

\begin{tabular}{|c|c|c|c|c|c|c|c|c|c|}
\hline \multirow{2}{*}{ Image } & \multicolumn{4}{|c|}{ ANM } & \multicolumn{3}{c|}{ Huffman Coder } & \multicolumn{3}{c|}{ Arithmetic Coder } \\
\hline $\begin{array}{c}\text { Image } \\
\text { Size }\end{array}$ & $\mathbf{5 1 2 * 5 1 2}$ & $\mathbf{2 5 6 * 2 5 6}$ & $\mathbf{1 2 8 * 1 2 8}$ & $\mathbf{5 1 2 * 5 1 2}$ & $\mathbf{2 5 6} * \mathbf{2 5 6}$ & $\mathbf{1 2 8 * 1 2 8}$ & $\mathbf{5 1 2 * 5 1 2}$ & $\mathbf{2 5 6 * 2 5 6}$ & $\mathbf{1 2 8 * 1 2 8}$ \\
\hline Lena & $134267 \mathrm{~B}$ & $33120 \mathrm{~B}$ & $8235 \mathrm{~B}$ & $133789 \mathrm{~B}$ & $32907 \mathrm{~B}$ & $8022 \mathrm{~B}$ & $132456 \mathrm{~B}$ & $66288 \mathrm{~B}$ & $8214 \mathrm{~B}$ \\
\hline Boat & $144563 \mathrm{~B}$ & $34856 \mathrm{~B}$ & $8486 \mathrm{~B}$ & $143189 \mathrm{~B}$ & $33112 \mathrm{~B}$ & $8355 \mathrm{~B}$ & $142123 \mathrm{~B}$ & $33275 \mathrm{~B}$ & $8261 \mathrm{~B}$ \\
\hline Man & $164260 \mathrm{~B}$ & $41410 \mathrm{~B}$ & $10254 \mathrm{~B}$ & $162456 \mathrm{~B}$ & $42795 \mathrm{~B}$ & $8216 \mathrm{~B}$ & $161763 \mathrm{~B}$ & $35487 \mathrm{~B}$ & $8419 \mathrm{~B}$ \\
\hline Satellite & $183277 \mathrm{~B}$ & $42934 \mathrm{~B}$ & $10444 \mathrm{~B}$ & $181234 \mathrm{~B}$ & $42145 \mathrm{~B}$ & $10469 \mathrm{~B}$ & $179845 \mathrm{~B}$ & $42188 \mathrm{~B}$ & $10289 \mathrm{~B}$ \\
\hline Medical & $194213 \mathrm{~B}$ & $48349 \mathrm{~B}$ & $11223 \mathrm{~B}$ & $192267 \mathrm{~B}$ & $44752 \mathrm{~B}$ & $11020 \mathrm{~B}$ & $190089 \mathrm{~B}$ & $42287 \mathrm{~B}$ & $10303 \mathrm{~B}$ \\
\hline Airplane & $154852 \mathrm{~B}$ & $33636 \mathrm{~B}$ & $8388 \mathrm{~B}$ & $154154 \mathrm{~B}$ & $33456 \mathrm{~B}$ & $8345 \mathrm{~B}$ & $154009 \mathrm{~B}$ & $33210 \mathrm{~B}$ & $8261 \mathrm{~B}$ \\
\hline House & $142475 \mathrm{~B}$ & $35225 \mathrm{~B}$ & $8734 \mathrm{~B}$ & $142123 \mathrm{~B}$ & $34471 \mathrm{~B}$ & $8603 \mathrm{~B}$ & $141475 \mathrm{~B}$ & $34193 \mathrm{~B}$ & $8527 \mathrm{~B}$ \\
\hline Baboon & $135647 \mathrm{~B}$ & $39510 \mathrm{~B}$ & $9854 \mathrm{~B}$ & $135231 \mathrm{~B}$ & $39247 \mathrm{~B}$ & $9736 \mathrm{~B}$ & $134756 \mathrm{~B}$ & $38600 \mathrm{~B}$ & $9633 \mathrm{~B}$ \\
\hline Pepper & $125647 \mathrm{~B}$ & $33701 \mathrm{~B}$ & $8413 \mathrm{~B}$ & $125142 \mathrm{~B}$ & $33546 \mathrm{~B}$ & $8378 \mathrm{~B}$ & $125035 \mathrm{~B}$ & $33374 \mathrm{~B}$ & $8325 \mathrm{~B}$ \\
\hline Bridge & $119685 \mathrm{~B}$ & $37036 \mathrm{~B}$ & $9240 \mathrm{~B}$ & $119254 \mathrm{~B}$ & $36760 \mathrm{~B}$ & $9183 \mathrm{~B}$ & $118254 \mathrm{~B}$ & $36178 \mathrm{~B}$ & $9000 \mathrm{~B}$ \\
\hline
\end{tabular}

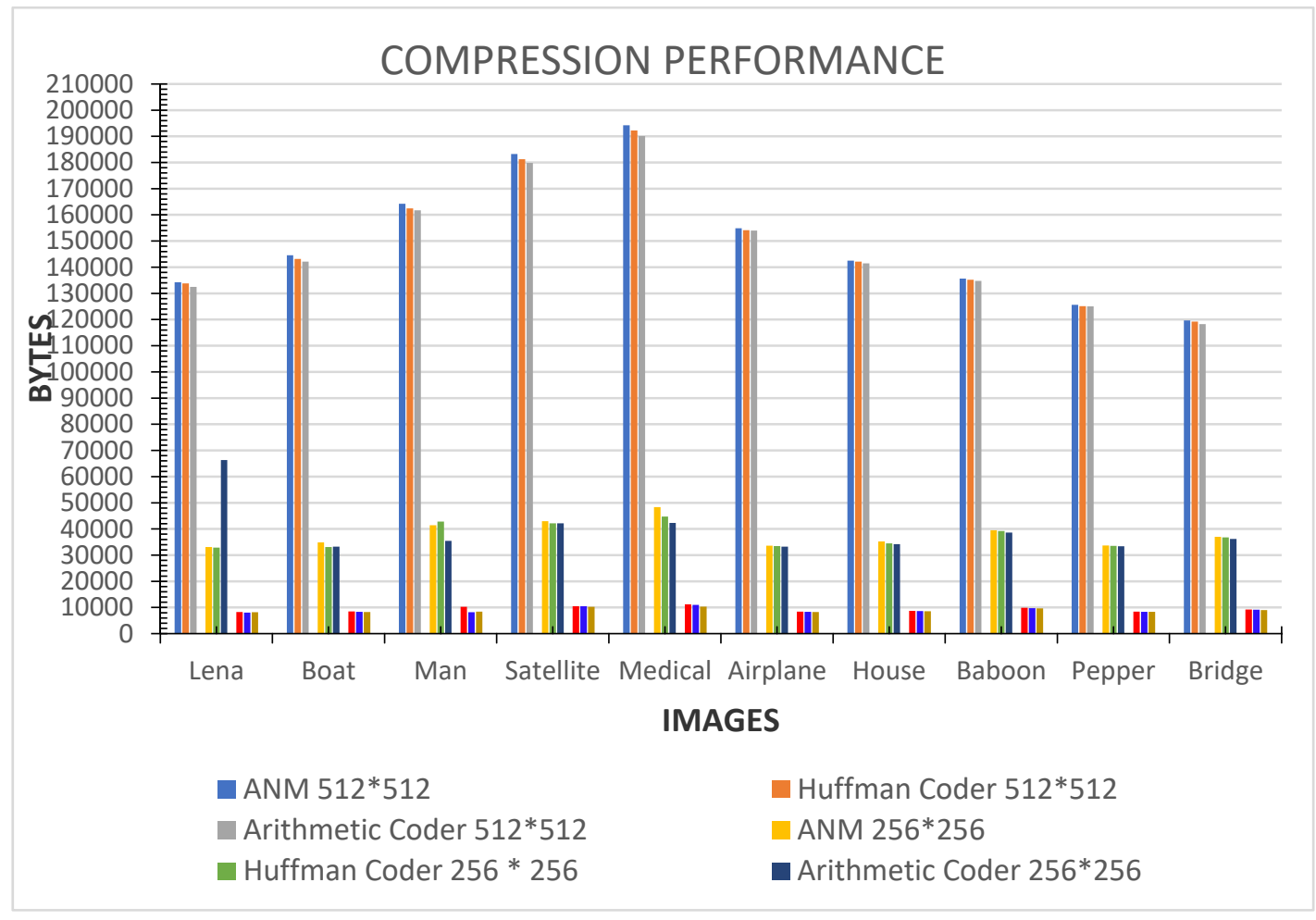

Figure.4 Compression Performance 


\section{Bits Per Pixel [BPP]}

BPP is defined as the number of bits of information stored per pixel of a given image. The more number of bits per pixel in an image ensures more number of colors can be represented but it increases the memory required to store and display the image. Table 2 shows comparison of compression ratio in Bits per Pixel (bpp) of proposed ANM with Huffman and Arithmetic coder. The Compression ratio for all the images is high when using ANM.

$$
\text { Bits per pixel }(b p p)=\left(\frac{\text { Size of compressed image in bits }}{\text { number of pixels }}\right)
$$

Table.2 Comparison of Compressed Ratio in Bits per Pixel [bpp]

\begin{tabular}{|c|c|c|c|c|c|c|c|c|c|}
\hline Image & \multicolumn{3}{|c|}{ ANM } & \multicolumn{3}{c|}{ Huffman Coder } & \multicolumn{3}{c|}{ Arithmetic Coder } \\
\hline $\begin{array}{c}\text { Image } \\
\text { Size }\end{array}$ & $\mathbf{5 1 2 * 5 1 2}$ & $\mathbf{2 5 6} * \mathbf{2 5 6}$ & $\mathbf{1 2 8 * 1 2 8}$ & $\mathbf{5 1 2 * 5 1 2}$ & $\mathbf{2 5 6} \mathbf{2 5 6}$ & $\mathbf{1 2 8 * 1 2 8}$ & $\mathbf{5 1 2} * \mathbf{5 1 2}$ & $\mathbf{2 5 6} \mathbf{2 5 6}$ & $\mathbf{1 2 8 * 1 2 8}$ \\
\hline Lena & 4.094 & 4.043 & 4.021 & 4.084 & 4.042 & 4.011 & 4.065 & 4.032 & 4.011 \\
\hline Boat & 4.411 & 4.255 & 4.144 & 4.345 & 4.017 & 4.080 & 4.125 & 4.062 & 4.034 \\
\hline Man & 5.112 & 5.055 & 5.007 & 4.922 & 4.042 & 4.012 & 4.754 & 4.322 & 4.111 \\
\hline Satellite & 5.593 & 5.241 & 5.100 & 5.456 & 5.224 & 5.112 & 5.645 & 5.150 & 5.024 \\
\hline Medical & 5.926 & 5.902 & 5.480 & 5.526 & 5.463 & 5.381 & 5.324 & 5.162 & 5.031 \\
\hline Airplane & 4.185 & 4.106 & 4.096 & 4.091 & 4.084 & 4.075 & 4.072 & 4.054 & 4.034 \\
\hline House & 4.325 & 4.300 & 4.265 & 4.214 & 4.208 & 4.201 & 4.194 & 4.174 & 4.164 \\
\hline Baboon & 4.851 & 4.823 & 4.812 & 4.804 & 4.791 & 4.754 & 4.738 & 4.712 & 4.704 \\
\hline Pepper & 4.127 & 4.114 & 4.108 & 4.103 & 4.095 & 4.091 & 4.085 & 4.074 & 4.065 \\
\hline Bridge & 4.547 & 4.521 & 4.512 & 4.507 & 4.491 & 4.484 & 4.413 & 4.409 & 4.395 \\
\hline
\end{tabular}

COMPRESSION RATIO [BITS PER PIXEL]

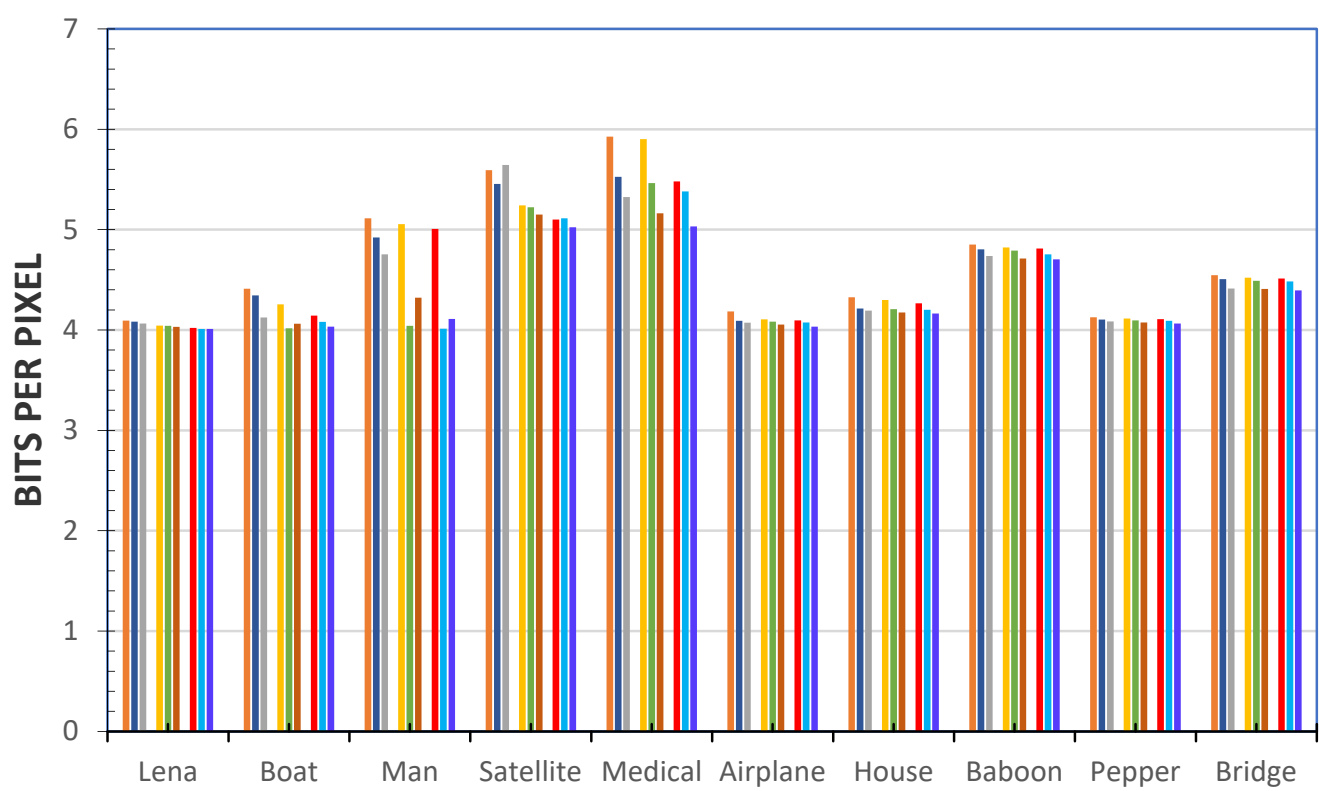

IMAGES

aNM $512 * 512$

- Arithmetic Coder 512*512

- Huffman Coder $256 * 256$
- Huffman Coder $512 * 512$

ANM 256*256

- Arithmetic Coder 256*256

Figure.5 Compression Ratio [BPP] 


\section{Computation Time}

Table 3 shows comparison of computation time of ANM. It is found that, ANM takes less computation time than Huffman coder and Arithmetic coder. From figure.6, It is observed that the ANM coder is faster than existing coders for image size $512 * 512$ and when the image size is increased there will be more efficiency.

Table 3 Comparison of Computation Time

\begin{tabular}{|c|c|c|c|c|c|c|c|c|c|}
\hline Image & \multicolumn{4}{|c|}{ ANM } & \multicolumn{3}{c|}{ Huffman Coder } & \multicolumn{3}{c|}{ Arithmetic Coder } \\
\hline Image Size & $\mathbf{5 1 2 * 5 1 2}$ & $\mathbf{2 5 6 * 2 5 6}$ & $\mathbf{1 2 8} * \mathbf{1 2 8}$ & $\mathbf{5 1 2 * 5 1 2}$ & $\mathbf{2 5 6} * \mathbf{2 5 6}$ & $\mathbf{1 2 8} * \mathbf{1 2 8}$ & $\mathbf{5 1 2 * 5 1 2}$ & $\mathbf{2 5 6 * 2 5 6}$ & $\mathbf{1 2 8 * 1 2 8}$ \\
\hline Lena & $2.7434 \mathrm{~s}$ & $1.7553 \mathrm{~s}$ & $0.7632 \mathrm{~s}$ & $2.7975 \mathrm{~s}$ & $1.7912 \mathrm{~s}$ & $0.8078 \mathrm{~s}$ & $2.8267 \mathrm{~s}$ & $1.8203 \mathrm{~s}$ & $0.8398 \mathrm{~s}$ \\
\hline Boat & $2.7569 \mathrm{~s}$ & $1.7743 \mathrm{~s}$ & $0.7825 \mathrm{~s}$ & $2.8234 \mathrm{~s}$ & $1.8302 \mathrm{~s}$ & $0.8487 \mathrm{~s}$ & $2.8567 \mathrm{~s}$ & $1.8601 \mathrm{~s}$ & $0.8745 \mathrm{~s}$ \\
\hline Man & $2.8755 \mathrm{~s}$ & $1.8867 \mathrm{~s}$ & $0.8854 \mathrm{~s}$ & $2.9043 \mathrm{~s}$ & $1.9156 \mathrm{~s}$ & $0.9250 \mathrm{~s}$ & $2.9479 \mathrm{~s}$ & $1.9467 \mathrm{~s}$ & $0.9500 \mathrm{~s}$ \\
\hline Satellite & $2.7743 \mathrm{~s}$ & $1.7832 \mathrm{~s}$ & $0.7902 \mathrm{~s}$ & $2.8065 \mathrm{~s}$ & $1.8189 \mathrm{~s}$ & $0.8254 \mathrm{~s}$ & $2.8343 \mathrm{~s}$ & $1.8467 \mathrm{~s}$ & $0.8513 \mathrm{~s}$ \\
\hline Medical & $2.8276 \mathrm{~s}$ & $1.8389 \mathrm{~s}$ & $0.8302 \mathrm{~s}$ & $2.8583 \mathrm{~s}$ & $1.8489 \mathrm{~s}$ & $0.8543 \mathrm{~s}$ & $2.8612 \mathrm{~s}$ & $1.8789 \mathrm{~s}$ & $0.8856 \mathrm{~s}$ \\
\hline Airplane & $2.845 \mathrm{~s}$ & $1.754 \mathrm{~s}$ & $0.856 \mathrm{~s}$ & $2.953 \mathrm{~s}$ & $1.785 \mathrm{~s}$ & $0.854 \mathrm{~s}$ & $2.984 \mathrm{~s}$ & $1.895 \mathrm{~s}$ & $0.854 \mathrm{~s}$ \\
\hline House & $2.754 \mathrm{~s}$ & $1.765 \mathrm{~s}$ & $0.845 \mathrm{~s}$ & $2.845 \mathrm{~s}$ & $1.754 \mathrm{~s}$ & $0.754 \mathrm{~s}$ & $2.798 \mathrm{~s}$ & $1.854 \mathrm{~s}$ & $0.896 \mathrm{~s}$ \\
\hline Babbon & $2.564 \mathrm{~s}$ & $1.865 \mathrm{~s}$ & $0.754 \mathrm{~s}$ & $2.756 \mathrm{~s}$ & $1.845 \mathrm{~s}$ & $0.654 \mathrm{~s}$ & $2.965 \mathrm{~s}$ & $1.985 \mathrm{~s}$ & $0.865 \mathrm{~s}$ \\
\hline Pepper & $2.684 \mathrm{~s}$ & $1.784 \mathrm{~s}$ & $0.754 \mathrm{~s}$ & $2.984 \mathrm{~s}$ & $1.954 \mathrm{~s}$ & $0.685 \mathrm{~s}$ & $2.854 \mathrm{~s}$ & $1.954 \mathrm{~s}$ & $0.754 \mathrm{~s}$ \\
\hline Bridge & $2.765 \mathrm{~s}$ & $1.756 \mathrm{~s}$ & $0.865 \mathrm{~s}$ & $2.854 \mathrm{~s}$ & $1.845 \mathrm{~s}$ & $0.984 \mathrm{~s}$ & $2.954 \mathrm{~s}$ & $1.954 \mathrm{~s}$ & $0.965 \mathrm{~s}$ \\
\hline
\end{tabular}

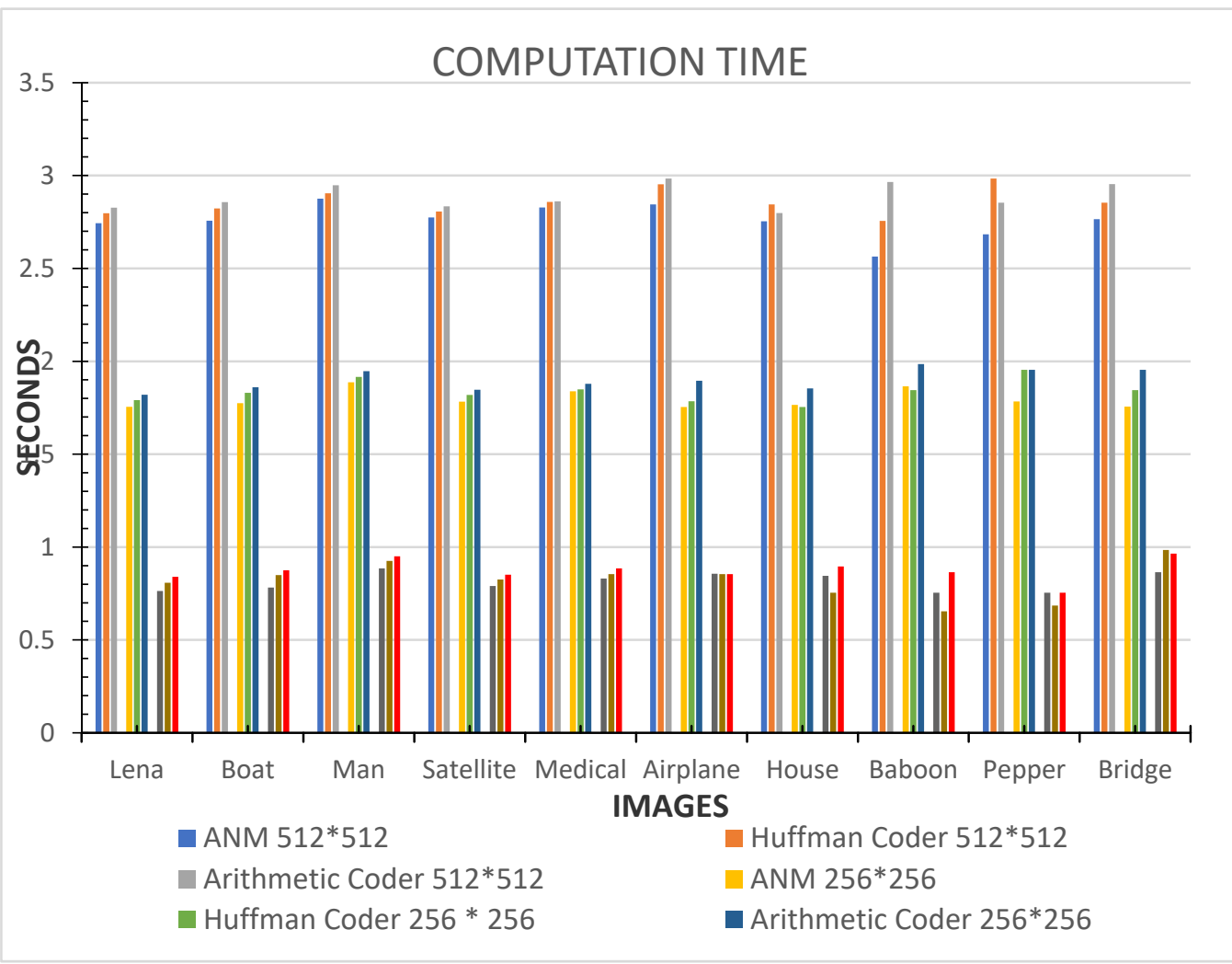

Figure.6 Computation Time

\section{Mean Square Error (MSE)}

The Mean Square Error (MSE) of an estimator measures the average of squares of the errors or deviations, that is, the difference between the estimator and what is estimated. MSE computes the average of squares of the errors from an image.

$$
M S E=\frac{1}{m n} \sum_{i=0}^{m-1} \sum_{j=0}^{n-1}[I(i, j)-K(i, j)]^{2}
$$


From table 4, it is found that the MSE value is high when using the Arithmetic and Huffman coder while it is significantly reduced when using ANM coder.

Table 4 Comparison of MSE

\begin{tabular}{|c|c|c|c|c|c|c|c|c|c|}
\hline \multirow{2}{*}{ Image } & \multicolumn{4}{|c}{ ANM } & \multicolumn{3}{c}{ Huffman Coder } & \multicolumn{3}{c|}{ Arithmetic Coder } \\
\hline Image Size & $\mathbf{5 1 2 * 5 1 2}$ & $\mathbf{2 5 6 * 2 5 6}$ & $\mathbf{1 2 8 * 1 2 8}$ & $\mathbf{5 1 2} * \mathbf{5 1 2}$ & $\mathbf{2 5 6} \mathbf{2 5 6}$ & $\mathbf{1 2 8} * \mathbf{1 2 8}$ & $\mathbf{5 1 2 * 5 1 2}$ & $\mathbf{2 5 6} \mathbf{2 5 6}$ & $\mathbf{1 2 8 * 1 2 8}$ \\
\hline Lena & 0.075 & 0.058 & 0.032 & 0.42 & 0.54 & 0.65 & 0.73 & 0.7 & 0.96 \\
\hline Boat & 0.087 & 0.045 & 0.031 & 0.44 & 0.53 & 0.62 & 0.72 & 0.85 & 0.95 \\
\hline Man & 0.087 & 0.065 & 0.098 & 0.41 & 0.58 & 0.64 & 0.62 & 0.83 & 0.97 \\
\hline Satellite & 0.094 & 0.067 & 0.054 & 0.35 & 0.44 & 0.58 & 0.68 & 0.8 & 0.95 \\
\hline Medical & 0.087 & 0.0034 & 0.023 & 0.34 & 0.41 & 0.52 & 0.69 & 0.82 & 0.87 \\
\hline Airplane & 0.084 & 0.098 & 0.099 & 0.21 & 0.32 & 0.46 & 0.52 & 0.83 & 0.96 \\
\hline House & 0.087 & 0.095 & 0.098 & 0.35 & 0.47 & 0.48 & 0.58 & 0.81 & 0.87 \\
\hline Baboon & 0.065 & 0.072 & 0.084 & 0.28 & 0.31 & 0.45 & 0.52 & 0.72 & 0.85 \\
\hline Pepper & 0.074 & 0.083 & 0.096 & 0.24 & 0.38 & 0.49 & 0.54 & 0.89 & 0.98 \\
\hline Bridge & 0.079 & 0.081 & 0.086 & 0.22 & 0.39 & 0.45 & 0.58 & 0.86 & 0.85 \\
\hline
\end{tabular}

Peak Signal To Noise Ratio (PSNR)

Peak Signal to Noise Ratio defines the ratio between the maximum possible power of a signal and the power of corrupting noise that affects the fidelity of its representation. The PSNR (in dB) is defined as:

$$
P S N R=10 . \log _{10}\left(\frac{M A X^{2}}{M S E}\right)
$$

From table 5, it is clear that the ANM coder yields better PSNR values when compared to the Arithmetic and Huffman coder.

Table 5 Comparison of PSNR (db)

\begin{tabular}{|c|c|c|c|c|c|c|c|c|c|}
\hline Image & \multicolumn{3}{|c|}{ ANM } & \multicolumn{3}{|c|}{ Huffman Coder } & \multicolumn{3}{|c|}{ Arithmetic Coder } \\
\hline Image Size & $512 * 512$ & $256 * 256$ & $128 * 128$ & $512 * 512$ & $256 * 256$ & $128 * 128$ & $512 * 512$ & $256 * 256$ & $128 * 128$ \\
\hline Lena & 54 & 52 & 53 & 48 & 45 & 42 & 38 & 36 & 34 \\
\hline Boat & 57 & 53 & 52 & 48 & 46 & 41 & 39 & 34 & 32 \\
\hline Man & 51 & 50 & 48 & 46 & 45 & 41 & 38 & 34 & 31 \\
\hline Satellite & 52 & 50 & 49 & 42 & 46 & 43 & 38 & 34 & 32 \\
\hline Medical & 58 & 57 & 51 & 50 & 46 & 42 & 39 & 31 & 30 \\
\hline Airplane & 53 & 51 & 49 & 42 & 41 & 38 & 36 & 35 & 31 \\
\hline House & 52 & 51 & 48 & 46 & 41 & 40 & 38 & 34 & 32 \\
\hline Baboon & 59 & 57 & 54 & 51 & 50 & 46 & 42 & 40 & 38 \\
\hline Pepper & 50 & 48 & 45 & 46 & 42 & 40 & 38 & 34 & 37 \\
\hline Bridge & 53 & 50 & 49 & 46 & 42 & 40 & 38 & 35 & 34 \\
\hline
\end{tabular}

\section{CONCLUSION}

In this paper, Asymmetric Numerical Method (ANM) is proposed to improve the compression ratio of images. The ANM provides better compression ratio than Huffman and Arithmetic coder. Similarly, the ANM achieves better results in terms of BPP, MSE, PSNR and computation time. The Asymmetric Numerical Method is an efficient lossless compression method which compresses the images without degrading the quality of the image and the original image can be recovered without any loss after decompression. Thus the proposed ANM coder improves the performance of image compression and also preserves the sharpness of the images. The results proved that ANM has the advantage to combine the compression ratio of arithmetic coder with the speed of Huffman coder. In this paper, the reasonable level of security has been retained by using permutation based image 
encryption method. In future, the encryption which is a vital process in the image processing can be considered to enhance the security of images.

\section{REFERENCES}

[1] Jarek Duda, 2014. Asymmetric numeral Systems: entropy coding combining speed of huffman coding with compression rate of arithmetic coding, arXiv:1311.2540V2[CS.IT]6, Jan 2014

[2] J. Duda,Optimal encoding on discrete lattice with translational invariant constrains using statistical algorithms, arXiv:0710.3861.

[3] J.Duda, Asymmetric numerical systems, arXiv: 0902.0271.

[4] J. Duda, Data Compression Using Asymmetric Numeral Systems, Wolfram Demonstration Project.

[5] Jiantao Zhou, Xianming Liu, Oscar C.Au and Yuan Yan, Jan 2014. Designing an efficient image encryption -Then-Compression System via Prediction Error clustering and Random Permutation, IEEE Transactions on information Forensics and security, Vol 9 No.1.

[6] Johnson, M., Ishwar, P., Prabhakaran, V., Schonberg, D. and Ramchandran, K., 2004. On compressing encrypted data. IEEE Transactions on Signal Processing, 52(10), pp.2992-3006.

[7] Klinc, D., Hazay, C., Jagmohan, A., Krawczyk, H. and Rabin, T., 2012. On compression of data encrypted with block ciphers. IEEE transactions on information theory, 58(11), pp.6989-7001.

[8] Lazzeretti, R. and Barni, M., 2008, August. Lossless compression of encrypted grey-level and color images. In Signal Processing Conference, 2008 16th European (pp. 1-5). IEEE.

[9] Xinpeng Zhang, X., Ren, Y., Feng, G. and Qian, Z., 2011, October. Compressing encrypted image using compressive sensing. In Intelligent Information Hiding and Multimedia Signal Processing (IIH-MSP), 2011 Seventh International Conference on (pp. 222225). IEEE.

[10] Yan, C. and Liu, X., 2009, July. Research on lossy compression technology about the encrypt coding image. In Environmental Science and Information Application Technology, 2009. ESIAT 2009. International Conference on (Vol. 1, pp. 374-377). IEEE.

[11] Zhang, X., Feng, G., Ren, Y. and Qian, Z., 2012. Scalable coding of encrypted images. IEEE Transactions on Image Processing, 21(6), pp.3108-3114.

[12] Zhang, X., Ren, Y., Shen, L., Qian, Z. and Feng, G., 2014. Compressing encrypted images with auxiliary information. IEEE transactions on multimedia, 16(5), pp.1327-1336.

\section{AUTHOR PROFILE}

Ms. P.Sridevi received the Bachelor of Science in Chemistry from Vellalar Arts and Science College, Erode, India . She has completed her Master of Computer Applications and Master of Philosophy in Computer Science under Bharathidasan University. She has received Master of Science in Chemistry from Periyar University and Bachelor of Education (B.Ed.) from Bharathiar University. She is presently a Ph.D., Research Scholar, Department of Computer Science, Vellalar College for Women under Bharathiar University, Coimbatore, India. Her research interest is Image Processing .

Dr. J. Suguna received the master's degree in mathematics from Annamalai University, Chidambaram in 1988 and the Ph.D. degree in Computer Science from the Bharathiar University, Coimbatore in 2009. She is currently an Associate Professor with the Department of Computer Science, Vellalar College for Women (Autonomous), Erode, Tamil Nadu. Her research interests are AI, Data Mining, Text Mining and Image Processing. She is the author or co-author of over 30 publications in journals, conference proceedings and book chapters. She has presented a paper in an International Conference held at Cincinnati University, Cincinati, Ohio, USA. She has produced over 18 M.Phil. Scholars in Computer Science and guiding 8 Ph.D. Scholars at present. 